OBSERVATIONS

oN

THE OMENTAL SACS

WHICH ARE SOMETIMES FOUND IN

\title{
STRANGULATED HERNIA,
}

COMPLETELY ENVELOPING THE INTESTINE, WITH CASES AND DISSECTIONS.

TO WHICH HAS BEEN ADDED,

A TABLE OF ALL THE STRANGULATED HERNIE OPERATED ON AT ST. GEORGE'S HOSPITAL, IN 1842-43.

By PRESCOTT HEWETT; Esq., Curator of st. george's Pathological museum.

READ MAY 14TH, 1844.

IN an operation for strangulated hernia, the intestine is not unfrequently found surrounded by omentum, which, at first, appears to form a second sac; with a little care, however, the omentum may be unfolded, and the intestine thus easily laid bare. But the cases in which the intestine is contained in a complete sac, formed by the omentum, which it is absolutely necessary to divide, to reach the gut, have, it appears, been rarely met with. Few opportunities have therefore been afforded for examining, either the nature of these sacs, or the difficulties to which they may give rise, in an operation. 
That these sacs may, in an operation for strangulated hernia, become the source of very great difficulties, is proved by the cases and dissections which follow these observations; and by the subjoined quotations, from two of the first authorities on hernia, it will be seen how these difficulties have hitherto been considered.

" It is stated, but I have never seen it, that the omentum is sometimes the cause of stricture, by enveloping the intestine, and becoming thickened around it. Such an occurrence is possible, and ought to be looked for, and, if found, the stricture will be easily divided."*

"Instances have occurred, in which the omentum has formed a complete bag, including a portion of intestine: in such a case it must be sufficiently divided to expose the gut." $\dagger$ This author does not mention any case of his own, but quotes Richter, and adds, that two cases related by Mr. Hey seem to have been, in some respects, of this kind.

On referring to the cases related by $\mathrm{Mr}$. Hey, it will be seen, that in neither of them can it be said that the omentum formed a complete bag, including a portion of intestine; in the first case, $\ddagger$ the omentum appears to have been attached to the ring, and to the bottom of the sac, lying over the intestine, and surrounding it: there was no necessity for $d i$ viding this membrane to reach the intestine, which

\footnotetext{
* Sir A. Cooper, fol. edit., 1804, p. 31.

$\uparrow$ Mr. Lawrence, fifth edit., p. 309.

$\ddagger$ Hey, Pract. Observ. 3rd edit., p. 214.
} 
was laid bare by simply lifting up the omentum. In the second case,* omentum only was found in the hernial sac, to which it was adherent ; no aperture could be found leading into the abdomen : the sensations of the patient led to the supposition that the gut had slipped up at the time of the operation; but this is mere conjecture.

Richter $\dagger$ even does not mention any cases of these omental sacs, which he merely states exist sometimes. He advises that, in such cases, Ledran's directions, of making an incision in the anterior part of the omentum, and then reducing the parts, should be followed; but such a case, adds Richter, occurs, most probably, very rarely.

In neither of these authors is it mentioned that the neck of these omental sacs may sometimes become the sole cause of strangulation.

These sacs have been found in the three most common forms of hernia; but it is in the umbilical hernia that they have been generally observed; the relative situation of the intestine and the omentum, in the abdominal cavity, will easily explain this fact.

Complete omental sacs were found in four cases out of thirty-four operations for strangulated hernia, performed at St. George's Hospital in 1842-43; of these four cases, two were femoral, one inguinal, and one umbilical. The formation of these sacs is attributed, by Richter, to the firm agglutination of

* Hey, Pract. Observ., 3rd edit., p. 217.

$\uparrow$ Traité des Hernies, p. 133. 
the margins of the omentum which has surrounded the bowel. To this explanation of Richter's, which does not appear to be applicable to the majority of cases, the two following explanations of the manner in which these sacs are, in some cases, formed, have been added.

1st. The gut, completely enveloped by the omentum, passes through the ring, and the omentum thus disposed round the intestine, becomes attached to the circumference of the neck of the hernial sac ; this omental pouch is subsequently distended by the intestine, and thus forms a complete lining to the hernial sac.

2nd. An epiplocele takes place, and the portion of omentum which is protruded becomes altered in structure, and its folds firmly united to each other by the effusion of lymph; but, within the abdominal cavity, in the neighbourhood of the ring, the folds into which the omentum has been drawn may not be agglutinated; they will thus leave spaces into which a knuckle of intestine may insinuate itself, pass through the ring, and form for itself a bed in the altered mass of omentum which is in the hernial sac. It may happen, that two or three portions of gut may slip into the Gifferent spaces left between the folds of the omentum, and subsequently form for themselves separate pouches. Several separate sacs, with narrow necks, may be thus found in the omental mass which is in the hernial sac.

Once formed, these sacs may attain an immense size. In Case 4, the sac measured six inches in 
length, and eleven inches in circumference, at its broadest part.

The omentum in which a sac has been formed may, in the course of time, especially if it is irreducible, become altered in structure, either by the effusion of lymph, or by a deposition of fat which takes place in the walls of the sac.

By this alteration of structure the thickened sac may, in an operation, become the source of very great difficulties. In Case 4, it will be seen that so great was the accumulation of fat in the layers of the omentum forming the sac, that its walls were, in some places, more than an inch thick. In this case the deposition of fat had principally taken place towards the lower part of the sac; at its neck the omentum was but slightly altered in structure. The incision made at the time of the operation corresponded to this part of the omentum, so that, when the hernial sac was laid open, the gut appeared to be covered merely by a band of omentum, which was easily divided, and the gut reduced; after which the sac was ascertained to be, with the exception of this slightly thickened band of omentum, quite empty; but no satisfactory explanation could be given of the nature of the thickened mass which still remained in the scrotum. A careful dissection, made at the post-mortem examination, proved that this mass consisted of the thickened omental sac, above referred to.

These omental sacs may either lie loose in the cavity of the hernial sac, or the two sacs may have 
contracted more or less extensive and firm adhesions with each other. In Case 3, the omental sac was quite free from adhesions; in Case 2, the two sacs adhered firmly to each other at the neck, in the whole of their circumference; and in Case 4, the two sacs were firmly united to each other, throughout their whole extent, by a fine, close, cellular tissue.

The above cases naturally give rise to a question of great practical importance, as to what course ought to be pursued where the hernial sac appears to contain thickened omentum only. In such cases the omentum ought to be drawn out and carefully examined, to see that it does not form a sac containing a portion of intestine. If the omentum is thickened, and firmly united to the neck of the hernial sac, throughout its whole circumference, a longitudinal incision should be carefully made in the whole length of the thickened omentum, to ascertain that it does not form one of these omental sacs. The enormous thickness which the walls of an omental sac may present, must here be borne in mind. The precaution of introducing the finger to ascertain that the neck of the sac is free, is, in these cases, particularly necessary.

The intestine contained in an omental sac may be united to its internal surface. In Case 1, the intestine was firmly united to the neck of the omental sac; in the three other cases, the intestine was free from adhesions.

The neck of an omental sac may become the sole 
cause of strangulation: of this, Case 3 is a wellmarked example. In this case, Mr. Hawkins was obliged, after having freely divided the neck of the hernial sac and the ring, to divide the neck of the omental sac before the gut could be reduced. Had the practice of reducing the hernia, without opening the hernial sac, been followed in this case, the gut, still strangulated by the omental sac, might have been reduced, and a fatal termination been the consequence. Such cases as these are a strong argument against the practice of reducing a hernia without opening the hernial sac.

The division of the neck of these omental sacs may be followed by hæmorrhage, as in Case 2 . The arteries of a portion of omentum which is hypertrophied and altered in structure, are sometimes so much enlarged that they may become a source of alarming hæmorrhage. Richter mentions a case in which an enlarged omental vessel poured out about $\zeta v j$ of blood in a few seconds. Particular attention should be paid to this point of practice, as the greater part of the blood may, from the situation of the divided vessel, at the neck of the sac, be poured into the cavity of the abdomen. In the above-mentioned Case, No. 2, the hæmorrhage, which occurred after the division of the neck of the omental sac, was but slight, and soon stopped ; but at the postmortem examination, a large patch of blood was found in the folds of the omentum, within the abdominal cavity, in the neighbourhood of the ring.

In conclusion, the occurrence of these omental 
sacs points out the importance of carefully examining every portion of omentum which is in a hernial sac, so as to ascertain that no knuckle of intestine is contained within its folds, before it is returned into the abdomen, left in the sac, or removed altogether.

Case 1.-Bowel contained in a complete sac formed by the omentum.

Maria Snell, æt. 60, was admitted into St. George's Hospital, under the care of Mr. Cutler, on the 28th of December 1843, at 5 P. M., with symptoms of strangulated umbilical hernia: the symptoms, however, were not very urgent. She had been subject to hernia for the last thirty years, for which she had worn a truss, until within the last five or six years, when the hernia became irreducible, and then increased in size; from that time she has been subject to habitual constipation, accompanied by colic, and occasionally by inflammation of the bowels. Four days ago, after a fit of coughing, the tumour suddenly increased in size; the symptoms of strangulation came on on the first day, and have been gradually increasing.

Purgative medicine, a warm bath, and the taxis, were tried in vain; and, five hours after the admission of the patient, Mr. Cutler, finding that the symptoms were increasing, determined upon operating.

The sac, which was very thin and adherent to the skin at the anterior part, was laid open: in it was contained a portion of the large intestine, enveloped 
by the omentum, which formed a complete bag; these parts were firmly adherent to the umbilical ring at the right side : they were but slightly altered in colour. The stricture and neck of the sac were divided, and a portion of gut reduced; the omentum and portion of adherent intestine were left in the sac.

The symptoms of strangulation were relieved by the operation; but the following morning, peritonitis, of a low character, made its appearance. She was put upon calomel, with salines and laudanum every four hours. The symptoms of peritonitis went on increasing, and she died thirty-seven hours after the operation.

Examination 56 hours after death.-The skin of the umbilical region was, at the anterior part, closely adherent to the hernial sac, which contained a large portion of omentum and about four inches of the transverse colon; the omentum, in which a large quantity of fat had been deposited, formed a second sac, completely enveloping the intestine, which was partially but firmly united to it; the omental sac was adherent to the right and inferior part of the ring, which was quite free at the left and superior part. The gut and omentum contained in the sac presented but slight marks of increased vascularity. The cavity of the peritoneum contained some darkcoloured fluid. The small intestines and the right portion of the large intestine, not contained in the hernial sac, were enormously distended with flatus, the left portion of the large intestine was contracted. 
The cæcum and ascending colon were, in many places, united to the convolutions of the small intestine by patches of recently-effused lymph : these patches, which corresponded to similar ones on the mucous surface of the large intestine, were surrounded by a minute net-work of vessels ; there were no ulcerations. The other portions of the intestinal canal presented nothing remarkable.

Case 2.-Bowel contained in a complete omental sac.

Elizabeth Baker, æt. 65, was admitted into St. George's Hospital, under Mr. Tatum, on the 3rd of June 1843, at 1 A.M., with a strangulated femoral hernia, on the left side; all the symptoms of strangulation, which had existed about three hours, were well marked. She had been subject to hernia for some years. The taxis, which had been previously applied by a surgeon, was again tried by the house-surgeon, but unsuccessfully.

Mr. Tatum operated an hour after the patient's admission. The sac was laid open, and in it were found a portion of omentum and a knuckle of small intestine. The omentum, which formed a complete sac enveloping the intestine, was so adherent to the neck of the hernial sac that a director could not, at any point, be passed between these parts; the omental sac was therefore laid open, and the stricture and both sacs were then divided, after which slight hæmorrhage occurred, but it soon stopped; the gut, of a darkish colour, was easily reduced; the omentum 
was removed, after the application of two ligatures, each embracing one half of the whole mass.

All the symptoms of strangulation were relieved by the operation; the bowels acted copiously after a dose of castor oil. Towards the evening she was very feverish, and shortly afterwards became perfectly comatose, in which state she continued until she died, about twenty-four hours after the operation.

Examination twenty-four hours after death.-Extensive peritonitis, accompanied by an effusion of a slightly plastic character. Theomentum, which passed through the left femoral ring, was firmly adherent to the whole circumference of the neck of the hernial sac; here, in its folds, were the remains of the pouch which had contained the bowel, and a large patch of recently-effused blood; the pouch was traced into the hernial sac, where the remaining portion of the omentum, with the two ligatures which had been applied, was found. The portion of bowel which had been strangulated presented nothing but a slight discolouration.

Three or four small oval tumours, of an encephaloid nature, and connected with the left side of the falx, were found pressing upon the brain. In the left optic thalamus were the remains of an old apoplectic effusion.

It was subsequently ascertained that this patient had had, six months before her admission into the hospital, an attack of apoplexy, followed by hemiplegia of the right side, from which she had nearly recovered when the gut became strangulated. 
Case 3.-Bowel strangulated by the neck of an omental sac.

Mary Canty, æt. 35, admitted into St. George's Hospital, under Mr. Hawkins, on the 20th of March 1842, at 8 P.M., with urgent symptoms of strangulated hernia. The rupture, which occupied the right femoral ring, had been down four days, since which time the symptoms had been gradually increasing; the taxis had been applied, for some time, by a surgeon. She had been subject to hernia for the last three months, but had always, hitherto, been able to reduce it without difficulty, and had never worn a truss. Bleeding, a warm bath, and the taxis, were again tried, but without success.

Mr. Hawkins operated, an hour after the admission of the patient. The sac, which was very thin, was laid open, and in it was found a large mass of omentum, somewhat altered in structure. The neck of the sac and Gimbernat's ligament were divided, so that the finger could be easily introduced into the abdomen. The omentum was then carefully examined, and a knuckle of small intestine, of a very dark colour, was seen through its folds, which formed a complete sac; this omental sac was laid open, but before the intestine could be returned, it was found necessary to divide the neck of this second sac, which formed a very tight stricture; the intestine was then easily reduced, but the omentum was kept in the hernial sac.

The symptoms were relieved by the operation, 
but on the following day she had a sharp attack of peritonitis, which was treated by leeches, calomel and opium, and salines. On the third day after the operation, some fæculent discharge was passed by the wound; this discharge increased, and continued for five days, after which it ceased. The wound then put on a healthy appearance, and was completely healed within a month after the operation.

Case 4.-Bowel contained in a large sac, with parietes of an inch thick, formed by omentum hypertrophied and altered in structure.

Charles Emmett, æt. 42, admitted into St. George's Hospital, under Mr. Cutler, on the 3rd of April 1843, with a strangulated scrotal hernia, on the right side. The tumour, of an enormous size, very heavy, hard and smooth, was pyriform, and appeared to contain a large quantity of fluid: the testicle was evidently at the lower part. The symptoms of strangulation had existed three days. He had been subject to hernia, which hitherto had caused him but little inconvenience, for the last three years: had never worn a truss.

Bleeding, a warm bath, and the taxis, were tried, but without any effect. As the symptoms were increasing, Mr. Cutler operated. The stricture was divided, and an attempt made to reduce the hernia without opening the sac, but unsuccessfully: the sac, which was very thick, was then laid open, and about a pint and a half of bloody fluid escaped. The gut, of a very dark colour, was covered by a band of omentum, slightly altered in structure, 
which adhered to some part of the sac. It was found necessary to divide this omental band before the gut, about five inches of the small intestine, could be reduced. With the exception of the band of omentum, the sac was ascertained to be quite empty; but this side of the scrotum still remained much larger and heavier than that of the opposite side. The testicle, of its natural size, could be easily felt at the lower part of the scrotum.

The symptoms of strangulation were relieved by the operation, but on the following day he was attacked by peritonitis, of a low character, for which he was put upon calomel and opium. The peritonitis went on increasing, and he died about thirtysix hours after the operation.

Examination 34 hours after death.-Abdomen tympanitic. The peritoneum was extensively inflamed, and covered with recently-effused lymph, slightly uniting the convolutions of the intestines to each other. The small intestine, near its termination, was of a dark mahogany colour; this discolouration existed in all the coats, but the parts were neither thicker nor softer than natural; the discoloured portion of intestine measured about five inches ; its corresponding mesentery was very much inflamed, and presented large dark patches, looking like effused blood.

A portion of omentum, not much altered in structure, but presenting a puckered appearance, was traced through the internal abdominal ring into the scrotum, where it became very much thickened, 
and thus formed the large tumour which had been observed during life. On laying open the tumour, from the scrotum up to the internal abdominal ring, it was found to consist of a large pouch, with thick parietes, in which the intestine had been lodged. At the time of the examination, the cavity of this pouch, which at the internal abdominal ring opened into the cavity of the peritoneum, was filled with serous fluid and large masses of lymph; its internal surface, presenting an irregular and convoluted appearance, was of a bright red colour, and covered, in many places, with thick layers of recently-effused lymph adhering to it. This pouch was of a pearshape, with a very narrow neck, corresponding to the inguinal canal : it measured six inches in length, and eleven inches in circumference, at its broadest part; its parietes, more than an inch thick in many places, were completely formed by the folds of the omentum, in which large quantities of adipose tissue had accumulated; this accumulation of fat had, for the greater part, taken place below the neck of the pouch; at the neck, in which $\mathrm{Mr}$. Cutler had made his incision for releasing the strangulated intestine, the thickened omentum did not exceed a line and a half in depth. The outer surface of this enormous omental sac was quite smooth, and, below the constriction, adherent throughout to the hernial sac, which was very much thickened, and of a fibrous appearance; these two sacs had, towards the upper part, become so firmly united to each other, that they could not be separated: at the lower part the 
adhesions were not so firm. The thickened hernial sac was covered externally by a dense, fibrous membrane, which, at the lower part, was separated from it by a large quantity of cedematous cellular tissue ; this fibrous membrane appeared to be a condensation of the different fasciæ and neighbouring cellular tissue. The testicle, somewhat flattened, was adherent to the bottom of the external coat, described as being formed by a condensation of the fasciæ, \&c.; the cavity of the tunica vaginalis was nearly obliterated by extensive and close adbesions; the chord ran at the back part, between the peritoneal sac and the outer coat, to which the vas deferens was firmly attached in a great part of its length; the vessels were merely surrounded by loose cellular tissue.

Table of the Strangulated Hernice operated upon at St. George's Hospital, in 1842-43.

Number of Operations, 34.

Maximum, 97.* ${ }^{\text {Age. }} \quad$ Minimum, 25.

Sex.

Men, 17. Women, 17.

* The oldest patient, Louis Pouchie, stated that he was 107, but it was subsequently ascertained that he had made a mistake as to his age. 
Nature of the Hernia.

Inguinal, 14. One of which, a direct inguinal, occurred in a woman, and two were congenital.

Femoral, 18. Fuur of which occurred in men.

Umbilical, 2. Both occurred in women.

Period of its existence.

Maximum, 50 years (congenital). Minimum, 3 days.

\section{Situation.}

Right side, 23. Inguinal, 9. Femoral, 14.

Left side, 9. Inguinal, 5. Femoral, 4.

Umbilical, 2.

Period of strangulation.

Maximum, 7 days. Minimum, 3 hours.

Contents of the Sac.

Small Intestine, 29.

All were either inguinal or femoral.

Large Intestine, 4.

Cæcum....... 1. Inguinal, right side (with a knuckle of small intestine).

Transverse Colon, 2. One inguinal, left side (congenital). One umbilical.

Colon ....... 1. Inguinal, right side.

Omentum, 18.

Alone........ 2. One right femoral. One umbilical. With small Intestine, 13. Right side, 7. Inguinal 3, Femoral 4. Left side, 6. Inguinal 2, Femoral 4. With large Intestine, 3. One inguinal, right side. One inguinal, left side (congenital). One umbilical.

In 15 cases the omentum was found firmly adhering to various parts of the sac. In 1 case the omentum was behind the intestine. In 4 cases the omentum formed a complete sac, with a 
narrow neck, in which the bowel was contained: in 3 of these cases the omental sac was more or less adherent to the hernial sac.

\section{Result.}

$$
\text { Cured, 25. Died, 9.* }
$$

In 17 cases it was ascertained that no truss had ever been worn, and in 4 cases that the truss had not been worn lately. Of those where a truss had never been worn, the shortest period of the existence of the heruia was three days, and the longest period was 50 years (congenital).

In all the 34 cases the sac was opened; in some an attempt was made to reduce the heruia without opening the sac, but unsuccessfully.

In 3 cases the gut could not be returned into the abdomen on account of adhesions : in one of these the gut itself was united to the neck of the sac, and the sac was adherent to the skin. The patient, a man, recovered : he had been operated upon, on the same side, for strangulated hernia, in 1818. The other 2 cases proved fatal.

* The following appearances were observed in the post-mortem examinations of these cases:-1. Peritonitis; intestine darkcoloured and thickened; omentum adherent to the ring, and in a sloughy state; diffuse cellular inflammation behind the sac. 2. Peritonitis; intestine black, soft, and easily lacerable. 3. Peritonitis; intestine and omentum left in the sac on account of adhesions. 4. Peritonitis ; intestine dark-coloured, with patches of slate colour, and very soft; kidneys granular; pneumonia. 5. Peritonitis ; intestine slightly discoloured; uterus large and soft, with some remnants of sloughy placenta at its fundus. 6. Peritonitis; intestine mortified. 7. Slight peritonitis; inflammation of the mucous membrane of the intestine, in patches, most probably antecedent to operation; omentum and intestine left in the sac, on account of adhesions : these parts were but slightly altered in colour. 8. Peritonitis; intestine slightly discoloured; disease of brain. 9. Peritonitis; intestine of a dark mahogany colour, but neither thickened nor softened. 
The omentum was removed in 6 cases.* Five of these recovered; one died, comatose, a few hours after the operation (disease of the brain). sloughed in 2 cases. One recovered; one died. suppurated in 3 cases. All recovered.

The sac and subjacent cellular tissue suppurated in several cases, and in one case, which recovered, there was very extensive sloughing of the cellular tissue of the scrotum and abdomen.

* In some of the cases two ligatures, each embracing one half of the omental mass, were applied; in the other cases ligatures were applied to all the bleeding vessels. 